\title{
Knowledge and Competencies of Vaccinators on Routine Childhood Vaccination in Ghana
}

Daniel Ansong ${ }^{1}$, Francis Adjei Osei ${ }^{2}$, Anthony Enimil ${ }^{1}$, Kofi Boateng ${ }^{2}$, Isaac Nyanor ${ }^{3}$, Evans Xorse Amuzu ${ }^{3}$, Alfred Kwame Owusu ${ }^{4}$, and Nicholas Mensah Karikari² $^{2}$

${ }^{1}$ Department of Child Health, School of Medical Science, Kwame Nkrumah University of Science and Technology, Ghana

${ }^{2}$ Public Health Unit, Komfo Anokye Teaching Hsospital, Kumasi, Ghana

${ }^{3}$ Research and Development Unit, Komfo Anokye Teaching Hospital, Kumasi, Ghana

${ }^{4}$ Quality Assurance Unit, Komfo Anokye Teaching Hospital, Kumasi, Ghana

*Corresponding author: Francis Adjei Osei, Public Health Unit, Komfo Anokye Teaching Hsospital, Kumasi, Ghana, Tel: +233(0) 248168933; E-mail: francisph1@hotmail.com

Received date: April 16, 2018; Accepted date: May 05, 2018; Published date: May 12, 2018

Copyright: $@ 2018$ Ansong D, et al. This is an open-access article distributed under the terms of the Creative Commons Attribution License, which permits unrestricted use, distribution, and reproduction in any medium, provided the original author and source are credited.

\begin{abstract}
Background: The Expanded Programme on Immunization (EPI), since its inception in 1974, has helped avert about 2-3 million deaths annually. In Ghana, there is inadequate information on the transition from knowledge acquired in the classroom to practice among vaccinators. This study seeks to provide evidence on the level of knowledge and competencies of vaccinators in Ghana.
\end{abstract}

Method: A prospective cross-sectional study with consecutive sampling technique was employed to select 110 eligible subjects from health facilities in two districts in the Ashanti region of Ghana. Data were collected in real time using standardised tools, uploaded on the Open Data Kit (ODK), and subsequently exported to STATA 13.0 for statistical analysis.

Results: Seventy two percent $(72.73 \%)$ of respondents were from urban health facilities. Community Health Nurses constituted majority of respondents $83.09 \%$. Sixty-five percent $(65.46 \%)$ of participants had less than five years' experience in vaccination. BCG was correctly identified by $91.82 \%$ of respondents as a single dose vaccine and yellow fever by $89.09 \%$. Pentavalent, rotavirus and pneumococcal vaccine were correctly identified by $91.82 \%$, $82.73 \%$ and $82.73 \%$ respectively as multiple dose vaccines. Ninety seven percent $(97.27 \%)$ of respondents correctly mentioned pentavalent as having its first dose administered at 6 weeks. 98.18\% correctly indicated pentavalent and pneumococcal as given intramuscularly whereas $92.27 \%$ identified OPV and rotavirus as given orally. Eighty-eight percent $(88.18 \%)$ of respondents knew vaccine temperature charting is required twice a day.

Conclusion: The study revealed gaps in the principles of vaccine handling and administration. A critical need for routine assessment and refresher training for population where vaccination is conducted by different level of training.

Keywords: Knowledge; Competency; Vaccinators; Routine childhood vaccination; Ghana

\section{Background}

Historically, routine vaccination emanated from the success achieved through the small pox eradication initiative. In 1974, the
World Health Organisation (WHO) initiated the Expanded Programme on Immunisation (EPI) to sustain the gains made in prevention of the initial six diseases (tuberculosis, diphtheria, tetanus, pertussis, polio, and measles) [1]. In Ghana, EPI was launched in 1978, became operational in all 10 regions in 1985 and has since expanded it from the initial six antigens to 13 antigens. The EPI schedule in Ghana is summarised in Table 1.

\begin{tabular}{|c|c|c|}
\hline Antigen /Vaccine & Route of administration & Time of administration \\
\hline Baecille Calmette-Guerin(BCG) Oral Polio Vaccine 0 & intradermal injection Oral & At birth \\
\hline Penta-I, Pneumococcal-I, Rotavirus and OPV-I & Intramuscular injection Oral & 6 weeks \\
\hline Penta-II, Pneumococcal-II, Rotavirus and OPV-II & Intramuscular injection Oral & 10 weeks \\
\hline Penta-III, Pneumococcal-III and OPV-III & Intramuscular injection Oral & 14 weeks \\
\hline Measles-Rubella & Subcutaneous injection & 9 months \\
\hline
\end{tabular}


Table 1: Schedule of the Expanded Programme on Immunisation (EPI) in Ghana

Immunisation is known as one of the most successful, equitable and cost-effective public health intervention worldwide and more people than ever before are being reached with immunisation [1]. In 2011, an estimated 109 million children less than one year representing, eighty three percent $(83 \%)$ were vaccinated with 3 doses of DPT (DPT 3) vaccine, $84 \%$ (110 million) with measles and about $88 \%$ (114 million) with the BCG vaccine [2]. Immunisation currently averts an estimated 2-3 million death from Diptheria-Pertusis-Tetanus (DPT) and measles annually.

In the United States of America the debate on vaccine ethics has been advanced to encompass vaccine administration, and safety monitoring [3]. Insufficient access to vaccination services including vaccine information has been cited as one the key barriers in improving vaccinations in United State [4]. It is widely reported that immunisation is faced with several programmatic issues including Demographic factors such as difficulty in reaching out to target population, human factors such as poor motivation for vaccinators and insufficient knowledge and skills required of vaccinators. The various approaches in addressing the challenges regarding the immunisation programmes are widely captured in the WHO training modules for health workers and managers [5] which can be adopted and adapted by all WHO members states.

In order to sustain these benefits, it is important that vaccinators observe the standard practices before, during and after vaccination. WHO recommends that standard guidelines for immunisation in practice should ensure that before vaccination, immunisation worker have the capacity to identify the right patient at the right time, identify, prepare and administer the right vaccine(s) and correct dosages using the right cold chain practices [6]. Inspecting vaccine and diluent is critical in reducing adverse events following immunization. This is done by carefully examining the vaccine vial monitor (a label containing a heat-sensitive material which is placed on a vaccine vial to register cumulative heat exposure over time) to select the vaccine in a good state (preferable stages 1 and 2). Also it is important to ensure that cold chain system is maintained through regular monitoring of the refrigerator. The standard protocol recommends two times monitoring (Morning and afternoon) and recording the temperature on a chart. The type of vaccine determines the site of administration. The BCG vaccine is given on the right upper arm, measles and yellow fever given at the left and right upper arm respectively. Polio Vaccine is given orally and the pentavalent and pneumococcal vaccines at the anterolateral thigh.

During vaccination, vaccinators are to adhere to acceptable aseptic, injection safety and effective waste management practices. These include appropriate handling, use, and disposal of reconstitution and injection syringes. It also include aseptic procedures for the reconstitution of vaccines and handling

After vaccination, vaccinators must have in place and be familiar with procedures for managing clinical waste and Adverse Events Following Immunisation (AEFI). Additional capacities of vaccinators are required to ensure adherence to multi-dose vial policies for effective management of subsequent immunisation sessions. Furthermore, vaccinators are to ensure that the appropriate messaging and reminders are provided to care givers of vaccines to avert defaulters and enhance achievement of fully immunised status by the vaccines. In recent times, financing for immunisation has is faced with challenges resulting in a cutback in grants. The immunisation landscape is challenged with limited supply of vaccines and sustaining high cost of operational and cold chain cost $[7,8]$. It is therefore imperative for vaccinators to be knowledgeable and adhere to standard protocol in vaccine handling and administration in order to minimise wastage, build public confidence and sustain gains. Unlike drugs, the expectations from vaccinations are much higher and problems arising from vaccination are less acceptable to the general public. Vaccines are usually administered to healthy people including birth cohorts of infants and in vast numbers $[9,10]$. A high level of knowledge and a positive attitude to immunisation among healthcare workers are important determinants of achieving and maintaining high vaccine uptake.

Effective childhood vaccination includes but not limited to the selection of the appropriate target group of children and the appropriate site of administration to elicit the appropriate immune response. The selection and use of the correct vaccine, the correct route of administration and timing have been associated with professionalism of the health staff responsible [11,12]. In Ghana, public health nurses, community health nurses, midwives and disease control officers are responsible for childhood vaccination. The level of knowledge and competencies of these vaccinators have not been well explored in Ghana. This study, aimed at assessing the knowledge and competencies of vaccinators in the management of vaccines and its administration.

\section{Methods}

A prospective cross-sectional study was conducted at the Maternal and Child Health facilities in Kumasi Metropolis and the Atwima Nwabiagya district in the Ashanti Region of Ghana. It involved health workers whose routine activities involved vaccine management, handling and administration in the selected hospitals.

A consecutive sampling technique was employed to select 110 eligible subjects for the study. The sample size was estimated based on an anticipated $50 \%$ knowledge on vaccination practices among vaccinators and $95 \%$ confidence interval which correspond to 1.96 standard values, an alpha of $5 \%$ and a precision error of $10 \%$. The sample size took into account non-response rate of $10 \%$ and was rounded to 110 participants.

Data on the knowledge of EPI vaccines focused on timing and dosing of the vaccines while data on competencies focused on the site and route of administration as well as the technique for administering the vaccines.

Data was collected by trained research officers using the open data kit [ODK] electronic mobile data collection system on tablets. As part of consistency with regards to site of vaccine administration, anonymised images of a health professional administering vaccines were developed and used as visual aids. Respondents were required to point to the image that indicated their opinion of the appropriate site, 
Citation: Ansong D, Osei FA, Enimil A, Boateng K, Nyanor I, et al. (2018) Knowledge and Competencies of Vaccinators on Routine Childhood

Page 3 of 6

and technique for administering the specific vaccines in the Expanded Programme on Immunisation (EPI). These instruments were pretested using a similar study site which was not part of the site selected for the study and were validated based on the pre-test results.

The data was exported to STATA 13.0 (Standard Edition) for analysis. Basic summary statistics of socio-demographic variables and independent variables were conducted, and bivariate analysis of association between rural and urban differences in terms of knowledge on vaccination using chi square test was used.

Permission to conduct the study was obtained from the Committee on Human Research Publications and Ethics (CHRPE), of Kwame Nkrumah University of Science and technology and the Komfo Anokye Teaching Hospital in Ghana. Written informed consent was obtained from respondents to affirm their willingness to participate in the study.

\section{Results}

A total of 110 vaccinators were recruited in the study. Most of respondents $(72.73 \%, \mathrm{n}=80)$ were selected from health facilities in an urban setting. The respondents were community health nurses $83.64 \%$ $(n=92 / 110)$. More than half of respondents $(65.46 \%, n=72 / 110)$ had less than five years' experience as a vaccinator while 9 had more than ten years' experience (Table 2).

\begin{tabular}{|l|l|l|}
\hline Variable & Number (n=110) & Percentage \\
\hline Place of work & \multicolumn{2}{|l|}{} \\
\hline Rural & 30 & 27.27 \\
\hline Urban & 80 & 72.73 \\
\hline Occupation & \multicolumn{2}{|l|}{} \\
\hline Community Health Nurse & 92 & 83.64 \\
\hline Disease control officer & 13 & 11.82 \\
\hline Public health nurse & 2 & 1.82 \\
\hline Health assistant & 1 & 0.91 \\
\hline Nutrition Officer & 2 & 1.82 \\
\hline Years worked as a Vaccinator & \multicolumn{2}{|l|}{} \\
\hline$<5$ y & 72 & 65.46 \\
\hline $5-10$ y & 29 & 26.36 \\
\hline
\end{tabular}

\begin{tabular}{|l|l|l|}
\hline$>10$ yrs & 9 & 8.19 \\
\hline
\end{tabular}

Table 2: Demographic characteristics of respondents

Respondents were assessed on the principles of vaccination and routine antigen use with emphasis on the doses required by a child. For vaccines requiring single dose, BCG was identified by $91.82 \%$ and Yellow fever mentioned by $89.09 \%$ of the respondents. Pentavalent, Vitamin A, rotavirus and pneumococcal vaccine were mentioned by $91.82 \%, 87.16 \%, 82.73 \%$ and $82.73 \%$ respectively by respondents as vaccines/antigens that required multiple doses to elicit immune response (Figure 1). A further test to examine the rural and urban difference in terms of doses required to be administered showed no statistically significant difference in knowledge.

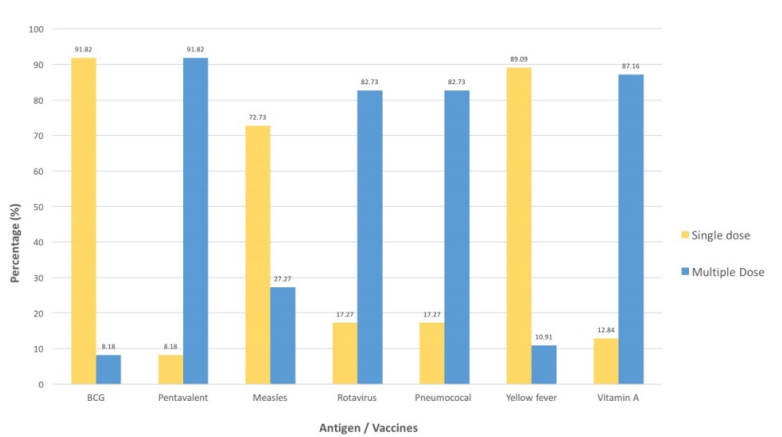

Figure 1: Knowledge on the Principles of Vaccination and Routine antigen use.

Respondents were also assessed on the time for administering the first dose of vaccines. All respondents indicated that BCG vaccine was given at birth. 107 (97.27\%) participants mentioned that Pentavalent administration starts at 6 weeks of a child's life. According to $91 \%$ of respondents $(n=101)$ the first dose of Vitamin A was given at 6 months. Also $97.27 \%$ and $91.82 \%$ respondents indicated that the first dose of yellow fever and measles respectively were given at 9 months (Table 3 ).

\begin{tabular}{|l|l|l|l|l|l|l|l|l|}
\hline \multirow{2}{*}{ Antigen/vaccine } & \multicolumn{9}{l}{ Time interval for administering vaccine, $\mathbf{n}(\%)$} \\
\cline { 2 - 10 } & At Birth & 6 weeks & 10 weeks & 14 weeks & 6 months & 9 months & 18 months & Don't known \\
\hline BCG & $110(100.00 \%)$ & - & - & - & - & - & - & - \\
\hline Pentavalent 1 & $1(0.91 \%)$ & $107(97.27 \%)$ & - & $1(0.91 \%)$ & - & - & - & $1(0.91 \%)$ \\
\hline Measles & - & $5(4.55 \%)$ & - & $1(0.91 \%)$ & $1(0.91 \%)$ & $101(91.82 \%)$ & $2(1.82 \%)$ & - \\
\hline Rotavirus 2 & $1(0.91 \%)$ & $108(98.18 \%)$ & - & - & - & $1(0.91 \%)$ & - & - \\
\hline Pneumococal 1 & - & $108(98.18 \%)$ & $1(0.91 \%)$ & - & $1(0.91 \%)$ & - & - & - \\
\hline Yellow fever & - & $1(0.91 \%)$ & - & - & $1(0.91 \%)$ & $107(97.27 \%)$ & $1(0.91 \%)$ & - \\
\hline
\end{tabular}


Citation: Ansong D, Osei FA, Enimil A, Boateng K, Nyanor I, et al. (2018) Knowledge and Competencies of Vaccinators on Routine Childhood Vaccination in Ghana. J Vaccines Vaccin 9: 389. doi:10.4172/2157-7560.1000389

Page 4 of 6

\begin{tabular}{|l|l|l|l|l|l|l|l|l|}
\hline Vitamin A & - & $7(6.36 \%)$ & - & - & $101(91.82 \%)$ & $2(1.82 \%)$ & - & - \\
\hline
\end{tabular}

Table 3: Time interval for administering routine vaccinations.

With a visual aid, majority $(98.18 \%, \mathrm{n}=108 / 110)$ of the respondents rightly indicated pentavalent and pneumococcal vaccines as given per intramuscular route at $90^{\circ}$ angle. About $69 \%$ rightly pointed out that BCG is given at an angle of $15^{\circ}$ intradermal. Majority $(97.27 \%$,
107/110) of the respondents correctly identified Vitamin A, OPV and Rotavirus as given through the oral route of administration (Table 4 and Table 5).

\begin{tabular}{|l|l|l|l|}
\hline Angle (Site) & vaccine & Responses \\
\cline { 3 - 4 } & & right & wrong \\
\hline $90^{\circ}$ (Intramuscular) & Penta/Pneumo & $108(98.18 \%)$ & $2(1.82 \%)$ \\
\hline $15^{\circ}$ (Intradermal) & BCG & $76(69.09 \%)$ & $34(30.91 \%)$ \\
\hline $45^{\circ}$ (Subcataneous) & Yellow fever/Measles & $89(80.91 \%)$ & $21(19.09 \%)$ \\
\hline Oral & Vit A/OPV/Rota & $107(97.27 \%)$ & $3(2.73 \%)$ \\
\hline
\end{tabular}

Table 4: Identification and recognition of appropriate sites/angles for administering routine vaccines.

\begin{tabular}{|c|c|c|c|c|}
\hline Antigen/vaccine & Rural, n (\%) & Urban, n (\%) & $x^{2}$ & p value \\
\hline \multicolumn{5}{|l|}{ BCG } \\
\hline Single Dose & $28(27.72 \%)$ & $73(72.28 \%)$ & 0.723 & 0.723 \\
\hline Multiple doses & $2(22.22 \%)$ & $7(77.78 \%)$ & & \\
\hline \multicolumn{5}{|l|}{ Pentavalent } \\
\hline Single Dose & $3(33.33 \%)$ & $6(66.67 \%)$ & 0.182 & 0.67 \\
\hline Multiple doses & $27(26.73 \%)$ & $74(73.27 \%)$ & & \\
\hline \multicolumn{5}{|l|}{ Measles } \\
\hline Single Dose & $25(31.25 \%)$ & $55(68.75 \%)$ & 2.339 & 0.126 \\
\hline Multiple doses & $5(16.67 \%)$ & $25(83.33 \%)$ & & \\
\hline \multicolumn{5}{|l|}{ Rotavirus } \\
\hline Single Dose & $6(31.58 \%)$ & $13(68.42 \%)$ & 0.215 & 0.643 \\
\hline Multiple doses & $24(26.37 \%)$ & $67(73.63 \%)$ & & \\
\hline \multicolumn{5}{|l|}{ Pneumococal } \\
\hline Single Dose & $5(26.32 \%)$ & $14(73.68 \%)$ & 0.011 & 0.918 \\
\hline Multiple doses & $25(27.47 \%)$ & $66(72.5 \%)$ & & \\
\hline \multicolumn{5}{|l|}{ Yellow fever } \\
\hline Single Dose & $28(28.57 \%)$ & 70 (71.43\%) & 0.764 & 0.382 \\
\hline Multiple doses & $2(16.67 \%)$ & $10(83.33 \%)$ & & \\
\hline \multicolumn{5}{|l|}{ Vitamin A } \\
\hline Single Dose & $5(35.71 \%)$ & 9 (64.29\%) & 0.54 & 0.462 \\
\hline
\end{tabular}

\begin{tabular}{|l|l|l|l|l|}
\hline Multiple doses & $25(26.32 \%)$ & $70(73.68 \%)$ & & \\
\hline
\end{tabular}

Table 5: Bivariate relationship between rural and urban regarding knowledge on the Principles of vaccination and routine antigen use.

The study further showed that vaccine temperature monitoring was done twice a day by $88.18 \%(\mathrm{n}=97 / 110)$ of the respondents (Figure 2$)$.

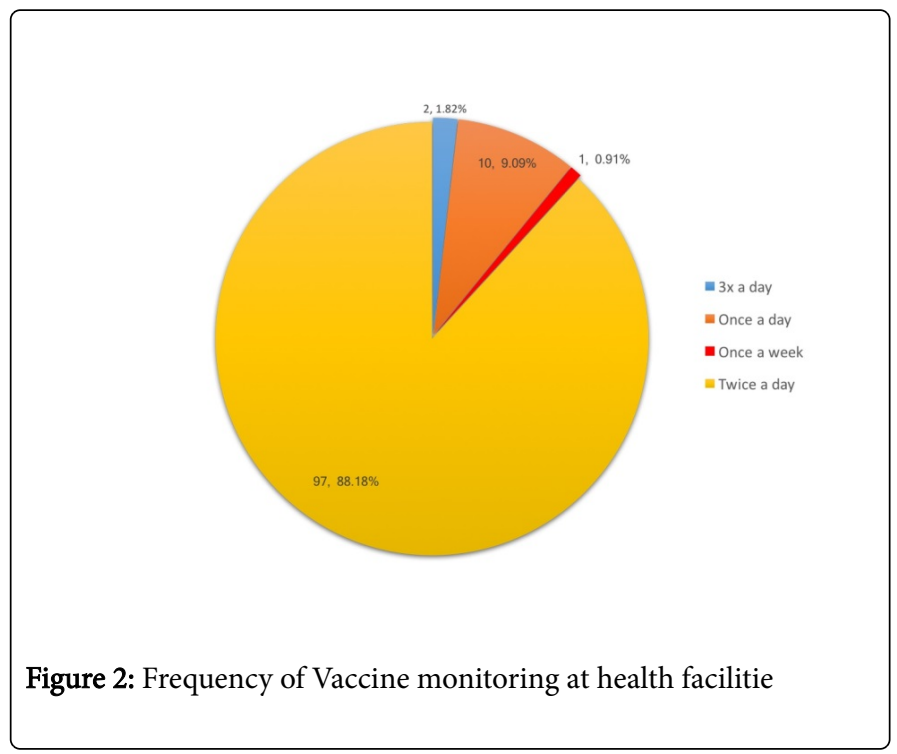

\section{Discussion}

The prime importance of vaccination is for the recipient to elicit the appropriate immunological response. Vaccine safety and effectiveness depend on several factors, which cumulatively lead to a successful vaccination corresponding to an immune response. Our study is of unique importance as the final pathway for vaccination programme depends on the knowledge and effective application of the skills of vaccinators. Health care managers assume that all vaccinators have the 
competencies required for a successful immunization of the child. It is worth noting that our study reveals that not all vaccinators who participated in the study knew the principles of vaccination and routine antigen use with emphasis on the doses required by a child and the time for administering the first dose of vaccines. In addition, a small percentage could not accurately identify the right angle for applying injections administration of some vaccines.

The right knowledge of vaccination by public and community health nurses who are on the forefront of vaccination programmes is key in addressing the shortfall in the information dissemination cycle for a successful immunization programme. The revelation of this study calls for a critical review of the training and refresher programmes available for vaccinators to ensure that all of the vaccinators are of equivalent level of knowledge regarding vaccination.

Secondly, identifying the right position for vaccination and injecting the right dose of vaccine is very important since it is directly linked to safety. Even though the percentage of vaccinators who could not rightly identify the position is small, it is a matter of concern since there are over thousands of vaccinators across the country and therefore If approximately $2 \%$ of the study population is extrapolated to the total number of vaccinators in the country then we potentially have large numbers of vaccinators administering vaccines inappropriately. Appropriately administering routine vaccines to children has been shown in a study by Kristensen et al. as a significant contributor to child survival [13]. The seeming difference between rural and urban could be as a result of strict adherence to vaccine protocol and supervision in urban areas. Vaccinators in the urban areas are more likely to undergo training on-the-job. It could also be as a result of the concentration of experienced vaccinators in urban areas. In addressing this gap, we advocate that the system should balance the cadre of experience across urban and rural settings.

Even though EPI Policy Guidelines, AEFI Policy Guidelines, Training modules including Immunisation in Practice and WHO MidLevel Management guidelines for vaccination practices exist in Ghana in addition to pre-training curriculum of Disease Control Officers and Community Health nurses, there are gaps in the systematic application of these policies and guidelines. Also, there are no policies available for certifying vaccinators and further monitoring and evaluating their competencies. This bring to fore a lapse in safety practices of administration of vaccines to children. It is worth noting that improper administration of vaccines may result in injuries or prevent the vaccines from providing optimal protection. In view of this, the Centre for Disease Control (CDC) recommends that all personnel required to administer vaccines should receive comprehensive, competency-based training regarding vaccine administration policies and procedures before administering vaccines. This is particularly tailored to new staff, hence the need to integrate it into new staff orientation programs. The CDC guidelines also recommends that providers of vaccination programs need to routinely validate staff's knowledge and competencies regarding vaccine administration through ongoing education for existing staff $[14,15]$. Evidence also suggests that simple and easily understood online materials in the form of audio-visuals can be used to address the knowledge gap. Studies by Johri et al. highlighted some sociological perspectives including the formation of peer-lead groups and use of Information, Communication and Technology such as the use of toll-free number system to enhance immunisation services [16].

Studies have shown that parents rely on healthcare professionals as their most trusted information source of vaccines, hence health professionals need to be ready to provide parents with timely and transparent information about vaccine benefits and risks. In our study it was reassuring to note that health education has been integrated into the vaccination program where mothers are routinely educated on the type of vaccine being received and the potential risks and benefits. This can be improved if there are programs designed to streamline the information given at all sites to minimise conflicting information. Information to caregivers/mothers are useful as this could influence vaccine acceptability and uptake [17]. This is even more critical for first time mothers. In a study by Anderson and Brown, first-time mothers were found to be more hesitant about vaccinations compared with mothers who already have children [18]. In this publication, effective communication between the vaccinator and the mother was associated with improvement in maternal knowledge in vaccination.

\section{Conclusion}

The study revealed gaps in the principles of vaccine handling and administration. It also highlights a critical need for routine assessment and re-training programme for vaccinators in routine immunization of different levels of training and experience. The Ghana Health Services should re-appraise the immunisation in practice capacities for health workers and develop regular professional refresher opportunities to keep them updated. In addition, renewal of professional certification by the relevance council and associations should include appraisals of professionals on their knowledge on vaccine handling and administration.

\section{Competing Interest}

None declared

\section{Funding}

There was no external funding for this study.

\section{Authors' Contributions}

Francis Adjei Osei, Daniel Ansong, Anthony Enimil and Kofi Boateng conceptualized and designed the study, trained data collectors, supervised and coordinated the data collection. They also drafted the initial manuscript, reviewed and revised the manuscript, and approved the final manuscript as submitted. Nicholas Karikari Mensah and Alfred Kwame Owusu conceptualized and designed the tools used for data extraction and collected the data. They also revised the manuscript and made inputs into the methodology. Isaac Nyanor and Evans Xorse Amuzu conducted data cleaning, analyzed the data and made inputs into the study design. All authors approved the final manuscript as submitted and agree to be accountable for all aspects of the work.

\section{Acknowledgement}

We wish to express appreciation to the respondents of this study.

\section{References}

1. Sentz, Mark A, Miller JT (2006) Disease and mortality in sub-Saharan Africa.

2. WHO (2013) Causality assessment of adverse event following immunization (AEFI): user manual for the revised WHO classification. Institutional Repos Inf Shar. 
Citation: Ansong D, Osei FA, Enimil A, Boateng K, Nyanor I, et al. (2018) Knowledge and Competencies of Vaccinators on Routine Childhood Vaccination in Ghana. J Vaccines Vaccin 9: 389. doi:10.4172/2157-7560.1000389

Page 6 of 6

3. Hendrix KS, Sturm LA, Zimet GD, Meslin EM (2016) Ethics and Childhood Vaccination Policy in the United States. Am J Public Health 106: 273-278.

4. National Cancer Institute T (2012) Accelerating HPV Vaccine Uptake: Urgency for Action to Prevent Cancer The President's Cancer Panel.

5. WHO (2015) Mid-Level Management Course for EPI Managers.

6. Hogue MD, Grabenstein JD, Foster SL, Rothholz MC (2017) Pharmacist involvement with immunizations: a decade of professional advancement. J Am Pharm Assoc 46: 168-182.

7. WHO (2015) Module 5: Managing an immunization session.

8. WHO (2011) Global Vaccine Action Plan: 2011-2020.

9. WHO (2014) Global Manual on Surveillance of Adverse Events Following Immunization.

10. WHO (2013) Global Vaccine Safety Initiative.

11. Salisbury D, Ramsay $M$, Noakes K (2013) Immunisation against infectious disease, Green Book 2006. Print Gt Britain by Station Off Updat 11: 1 .

12. MOH/GHS (2014) Immunization Programme Comprehensive MultiYear.
13. Kristensen I, Aaby P, Jensen H (2000) Routine vaccinations and child survival: follow up study in Guinea-Bissau, West Africa. Bmj 321: 1435-1438.

14. Centers for Disease Control and Prevention (U.S.) (2015) General recomendations on Immunization. Epidemiol Prev Vaccine-Preventable Dis 1: 9-32.

15. CDC (2017) Vaccine Administration.

16. Johri M, Chandra D, Koné GK, Dudeja S, Sylvestre MP, et al. (2015) Interventions to increase immunisation coverage among children 12-23 months of age in India through participatory learning and community engagement: pilot study for a cluster randomised trial. BMJ Open 5: e007972.

17. Angelillo I, Ricciardi G, Rossi P, Pantisano P, Langiano E, et al. (1999) Mothers and vaccination: knowledge, attitudes, and behaviour in Italy. Bull World Health Organ 77: 1.

18. Anderson S, Brown M (2017) First-time mothers more uncertain about child vaccinations, research shows. 